\title{
TITLE:
}

\section{Idea paper: Predicting culturability of microbes from population dynamics under field conditions}

$\operatorname{AUTHOR}(S)$ :

Ushio, Masayuki

\section{CITATION:}

Ushio, Masayuki. Idea paper: Predicting culturability of microbes from population dynamics under field conditions. Ecological Research 2020, 35(4): 586-590

\section{ISSUE DATE:}

2020-07

URL:

http://hdl.handle.net/2433/253576

\section{RIGHT:}

This is the peer reviewed version of the following article: Ushio, M. Idea paper: Predicting culturability of microbes from population dynamics under field conditions. Ecological Research. 2020; 35: 586- 590, which has been published in final form at https://doi.org/10.1111/1440-1703.12104. This article may be used for non-commercial purposes in accordance with Wiley Terms and Conditions for Use of Self-Archived Versions.; The full-text file will be made open to the public on 11 March 2021 in accordance with publisher's 'Terms and Conditions for Self-Archiving'.; This is not the published version. Please cite only the published version.; この論文は出版社版でありません。引用の際には出版社版をご硫認じ 利用ください。 
Ushio M, Microbial dynamics and culturability

10

11

12

13

14

15

16
Special Feature for Ecological Research

Running head: Microbial dynamics and culturability

Title:

Idea Paper: Predicting culturability of microbes from population dynamics under field conditions

\author{
Author: \\ Masayuki Ushio ${ }^{12,3^{*}}$
}

Affiliations of author:

'Hakubi Center, Kyoto University, Kyoto 606-8501, Japan, ${ }^{2}$ Center for Ecological Research, Kyoto University, Otsu 520-2113, Japan, sRESTO, Japan Science and Technology Agency, Kawaguchi 3320012, Japan

\section{*Corresponding author:}

Masayuki Ushio, Hakubi Center, Kyoto University, 606-8501 Japan, E-mail: ong8181@gmail.com, Tel: +81-77-549-8250, Fax: +81-77-549-8201 
Ushio M, Microbial dynamics and culturability

\section{Abstract:}

22 Isolation and cultivation of microbes from environmental samples have been

23 fundamental and important for species identification and investigating functions and

24 ecology of target microbes. While cultivation and isolation of microbes are not easy, the natural environment can "culture" any endemic microbes, and thus key information for culturing and isolating microbes may be encoded in the natural population dynamics of microbes. In the present paper, I present the idea that culturability of microbes may be inferred by quantifying dynamic properties of microbes using nonlinear time series analysis, empirical dynamic modeling (EDM). To briefly demonstrate the idea, I analyzed high-frequency, quantitative microbial time series obtained for experimental rice plots. I selected bacterial phyla that included sufficient numbers of microbial taxa, and analyzed 398 microbial taxa using empirical dynamic modeling. The nine phyla analyzed generally followed a similar pattern: many microbial taxa fell into the "Simple" dynamics category, and a small proportion of taxa were categorized in "Simple but nonlinear" or "Nearly random" dynamics categories. The present analysis suggested that many microbes in the study system might be cultivated by modifying a relatively small number of conditions. However, the present idea as well as the result is preliminary, and more precise taxonomic information (i.e., species-level identification) and a culturability dataset will help to validate the idea. If the present idea was found to be valid, a priori evaluation of the culturability of microbes would become possible, which would avoid unnecessary costs (labor, time and money) of attempts to cultivate microbes. 
Ushio M, Microbial dynamics and culturability

\section{Research question}

Isolation and cultivation of microbial species from environmental samples have been fundamental for identifying microbial species and investigating functions and ecology of a target microbial species. However, unfortunately, the majority of microbes in the environment cannot be cultured easily (e.g., Amann et al. 1995), and improving the recovery of microbes from environmental samples is an important but difficult and labor-, time- and money-consuming work. Therefore, if a priori evaluations of the culturability of microbes would become possible, it would contribute to avoiding unnecessary costs and improving the recovery of microbes from environments. For example, a cultivation design that includes a large number of combinations of nutrients in media might not be necessary if the target microbes were classified as "easily cultured".

\section{Value}

A priori evaluations of the culturability of microbes would contribute to reducing potential costs of cultivation. Also, a framework for quantifying culturability would enable analysis of the relationship between the culturability and genetic factors, which could potentially contribute to understanding why particular microbial species are difficult to culture while others are not.

\section{Relevant hypothesis (and approaches)}

While isolation and cultivation of microbes are not easy, natural environments can "culture" any endemic microbes, and thus key information for isolating and culturing microbes may be encoded in microbes' interactions with natural habitats and resultant population dynamics in nature (or under field conditions). Indeed, the diffusion chamber approach, which allows exchanges of chemicals between media and their natural habitat, can simulate the natural environment, and improves the recovery of some microbes from environment (Bollmann et al. 2007; Kaeberlein et al. 2002). These findings implied that microbes in nature respond to biotic/abiotic variables in their environment and the interactions among microbes and the environment drive their population dynamics. Therefore, conversely, natural population dynamics of microbes contain integrated information on biotic and abiotic variables and their interactions in the environment. The question is, however, how we can extract the information encoded in the population dynamics of microbes.

Information encoded in population dynamics can be extracted using empirical dynamic modeling (EDM) that is based on attractor reconstruction and designed for analyzing nonlinear dynamics (e.g., Sugihara et al. 2012). Core tools of EDM are the simplex projection (Sugihara and May 1990) and S-map (Sugihara 1994), which enable quantifying (1) the best embedding dimension and (2) state-dependence of time series data. Briefly, the best embedding dimension (denoted by $E$ ) includes information on how many potential variables might be involved in the process (i.e., complexity or dimensionality), and state-dependency (quantified by the nonlinear weighting parameter, $\theta$ ) includes information on how state dependent the process is. A previous study demonstrated that $E$ and $\theta$ are effective indices to distinguish random environmental fluctuations from low dimensional, nonlinear dynamics of 
Ushio M, Microbial dynamics and culturability

91

organisms (Hsieh et al. 2005). Detailed information on how simplex projection and Smap are performed is described in previous studies (Sugihara 1994; Sugihara and May 1990). Also, an overview of EDM is described in Chang et al. (2017).

\section{New research idea}

In the present paper, I present the idea that culturability of microbes may be inferred using population dynamics of microbes and EDM. Specifically, I expect that the complexity $(E)$ and state-dependency $(\theta)$ of population dynamics would provide information on the culturability of microbes.
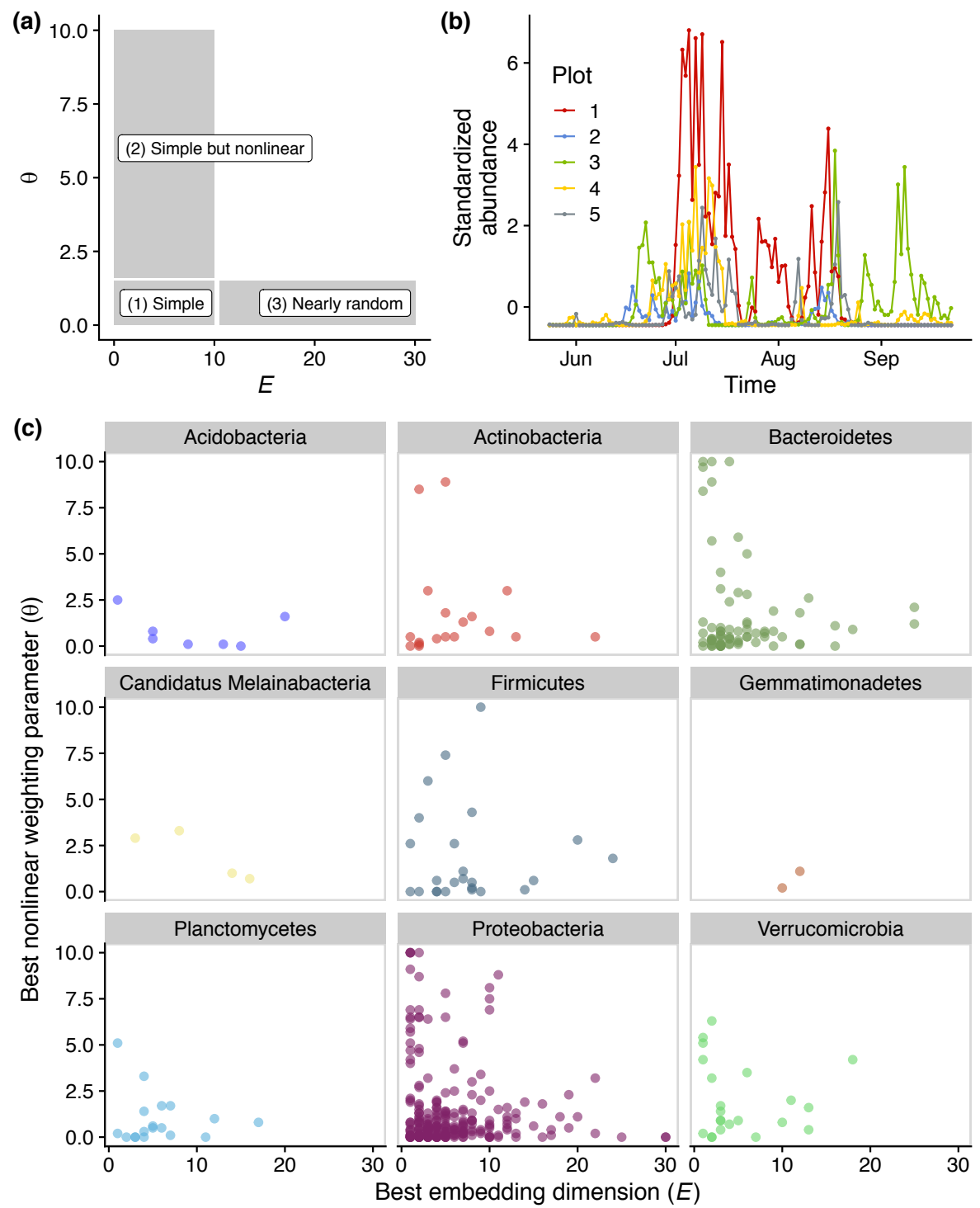

Figure 1 | (a) A conceptual explanation of the relationships between $E$ and $\theta$ and dynamics properties. Note that the thresholds between the categories are arbitrarily determined. (b) An example of time series analyzed in the present study. Changes in abundance (i.e., estimated DNA copy numbers) of a microbial amplicon sequence variant (ASV) belonging to Bacteroides are shown. Different colors indicate different rice plots where water samples were taken. (c) The relationships between $E$ and $\theta$ and microbial taxa. Each panel indicates a particular phylum, and each point indicates the best $E$ and $\theta$ of each ASV. 
Ushio M, Microbial dynamics and culturability

\section{How to answer the question through the research approach}

I expect three possible dynamics regarding the combinations of $E$ and $\theta$ by following Hsieh et al. (2005): (1) "Simple" (i.e., low dimensional dynamics), (2) "Simple but nonlinear", and (3) "Nearly random" dynamics (Fig. 1a). First, if the population dynamics of a target species show small $E$ and $\theta$, then the number of potential variables might be small and the species responds to cultivation conditions relatively linearly ("Simple" dynamics). In this case, the target microbe might be cultivated by examining a relatively small number of cultivation conditions. Second, if the population dynamics show small $E$ but large $\theta$, then the number of potential variables might be small but the growth of the species is state dependent ("Simple but nonlinear" dynamics). In this case, the target microbe could respond to cultivation conditions nonlinearly, and thus careful considerations of cultivation conditions would be required. Third, if the population dynamics show large $E$ but small $\theta$, then the dynamics are nearly random and the target microbe might have randomly immigrated from outside the system ("Nearly random" dynamics).

To briefly demonstrate the idea, I analyzed highly frequent, quantitative microbial time series taken from experimental rice plots established at the Center for Ecological Research, Kyoto University, Japan (Ushio, unpublished). Briefly, water samples were collected from five rice plots every day from 23 May to 22 September in 2017 (610 samples in total), and filtered using filter cartridges (pore size $=\phi 0.22 \mu \mathrm{m}$ ). Then, DNAs were extracted, amplified a portion of the 16S rRNA genes and sequenced using Illumina MiSeq. In the library preparation process, artificial standard DNAs with known concentrations were included to estimate the copy numbers of microbial DNAs (Ushio 2019). The sequences generated were analyzed using the amplicon sequence variant (ASV) approach (Callahan et al. 2016). I selected bacterial phyla that are abundant and contain sufficient numbers of ASVs, which resulted in 398 bacterial ASVs belonging to nine phyla. An example of the microbial time series is shown in Fig. 1b. For the time series selected, simplex projection and S-map were applied to determine the best $E$ and $\theta$ using rEDM packages (Ye et al. 2018) of R (R Core Team 2019). The whole dataset is being analyzed for different purposes and thus is currently not publicly available.

The nine phyla analyzed followed a similar pattern (Fig. 1c): many bacterial ASVs fall into the "Simple" category, and a relatively small proportion of ASVs were categorized as "Simple but nonlinear" or "Nearly random". This result might indicate that, among the selected bacterial ASVs, many microbes could be cultivated by modifying a relatively small number of conditions, which is contrary to the current general consensus that most environmental microbes are difficult to cultivate and isolate (Amann et al. 1995; but see Martiny 2019). However, technical issues (e.g., biases generated by an amplicon sequencing approach) may partly contribute to this pattern, and thus careful interpretations of the results are necessary (see debates in the following references: Martiny 2019; Steen et al. 2019).

Here I list data that are required to verify my idea more thoroughly. First, microbes should be identified at least at the species level. This is because the culturability would be a property of a species, and thus genus or family level identification is insufficient. The present data analyzed were generated by a shortread marker-gene (amplicon) analysis, and thus the phylogenetic resolution was 
Ushio M, Microbial dynamics and culturability

156 often not sufficient. Recently introduced long-read sequencing (e.g., full-length $16 \mathrm{~S}$

157 rRNA sequencing) would be more appropriate to achieve this goal (e.g., MinION by

158 Oxford Nanopore or Sequel by PacBio). Species-level dynamic properties would be

159 useful to classify and predict previously ignored aspects of microbial properties.

160 Second, accurate information on which microbial species and how the microbial

161 species is cultivated is required. Information on which microbial species is cultivated

162 would be available in public databases, but developing the cultivation method often

163 requires detailed tuning and thus the method as well as its complexity would be

164 difficult to fully document/digitalize. Compiling fundamental information about

165 cultivation methods (e.g., temperature and resources) would help to develop a

166 culturability database, which could also contribute to understanding of the potential

167 habitat range of microbes in nature. Together, if the present idea were validated by

168 overcoming the above limitations, dynamic properties of microbes could be linked to

169 culturability and a priori evaluation of the culturability of microbes would become

170 possible.

\section{Acknowledgements}

174 I would like to thank Asako Kawai for assistance in the sampling and experiments

175 and Hiroki Yamanaka for providing an opportunity to use Illumina MiSeq. This

176 study was financially supported by PRESTO (JPMJPR16O2) from the Japan Science and Technology Agency (JST) and the Hakubi project of Kyoto University.

Competing interests: The author has no competing interests. 
Ushio M, Microbial dynamics and culturability

182

183

184

185

186

187

188

189

190

191

\section{References}

Amann RI, Ludwig W, Schleifer KH (1995) Phylogenetic identification and in situ detection of individual microbial cells without cultivation. Microbiological Reviews 59:143-169

Bollmann A, Lewis K, Epstein SS (2007) Incubation of Environmental Samples in a Diffusion Chamber Increases the Diversity of Recovered Isolates. Applied and Environtal Microbiology 73:6386- 6390

Callahan BJ, McMurdie PJ, Rosen MJ, et al (2016) DADA2: High-resolution sample inference from Illumina amplicon data. Nature Methods 13:581-583

Chang C-W, Ushio M, Hsieh C (2017) Empirical dynamic modeling for beginners. Ecological Research 32:785-796

Hsieh C, Glaser SM, Lucas AJ, Sugihara G (2005) Distinguishing random environmental fluctuations from ecological catastrophes for the North Pacific Ocean. Nature 435:336-340

Kaeberlein T, Lewis K, Epstein SS (2002) Isolating Uncultivable; Microorganisms in Pure Culture in a Simulated Natural Environment. Science 296:1127-1129

Martiny AC (2019) High proportions of bacteria are culturable across major biomes. The ISME Journal 13:2125-2128

R Core Team (2019) R: A language and environment for statistical computing, Vienna, Austria

Steen AD, Crits-Christoph A, Carini P, et al (2019) High proportions of bacteria and archaea across most biomes remain uncultured. The ISME Journal 13:3126-3130

Sugihara G (1994) Nonlinear forecasting for the classification of natural time series. Philosophical Transactions of The Royal Society A: Mathematical, Physical and Engineering Sciences 348:477-495

Sugihara G, May R, Ye H, et al (2012) Detecting causality in complex ecosystems. Science 338:496-500

Sugihara G, May RM (1990) Nonlinear forecasting as a way of distinguishing chaos from measurement error in time series. Nature 344:734-41

Ushio M (2019) Use of a filter cartridge combined with intra-cartridge bead-beating improves detection of microbial DNA from water samples. Methods in Ecology and Evolution 10:1142-1156

Ye H, Clark A, Deyle E, et al (2018) rEDM: Applications of Empirical Dynamic Modeling from Time Series. doi:10.5281/zenodo.1935847 